University of New Hampshire

University of New Hampshire Scholars' Repository

Space Science Center

Institute for the Study of Earth, Oceans, and

Space (EOS)

1996

\title{
Using BATSE to measure gamma-ray burst polarization
}

\author{
Mark L. McConnell \\ University of New Hampshire - Main Campus, mark.mcconnell@unh.edu \\ D J. Forrest \\ University of New Hampshire - Main Campus \\ W T. Vestrand \\ University of New Hampshire - Main Campus \\ $\mathrm{M} \mathrm{H}$. Finger \\ Universities Space Research Association
}

Follow this and additional works at: https://scholars.unh.edu/ssc

Part of the Astrophysics and Astronomy Commons

\section{Recommended Citation}

Using BATSE to measure gamma-ray burst polarization McConnell, M. and Forrest, D. and Vestrand, W. T. and Finger, M., AIP Conference Proceedings, 384, 851-855 (1996), DOI:http://dx.doi.org/10.1063/1.51605

This Conference Proceeding is brought to you for free and open access by the Institute for the Study of Earth, Oceans, and Space (EOS) at University of New Hampshire Scholars' Repository. It has been accepted for inclusion in Space Science Center by an authorized administrator of University of New Hampshire Scholars' Repository. For more information, please contact Scholarly.Communication@unh.edu. 


\section{AIP $\mid$ Proceedings}

\section{Using BATSE to measure gamma-ray burst polarization}

M. McConnell, D. Forrest, W. T. Vestrand, and M. Finger

Citation: AIP Conference Proceedings 384, 851 (1996); doi: 10.1063/1.51605

View online: http://dx.doi.org/10.1063/1.51605

View Table of Contents:

http://scitation.aip.org/content/aip/proceeding/aipcp/384?ver=pdfcov

Published by the AIP Publishing

\section{Articles you may be interested in}

A Comparison of Gammaray Burst Subgroups Measured by RHESSI and BATSE AIP Conf. Proc. 1085, 674 (2008); 10.1063/1.3076765

BATSE observations of gamma-ray burst tails

AIP Conf. Proc. 526, 385 (2000); 10.1063/1.1361567

On the distribution of BATSE gamma-ray bursts

AIP Conf. Proc. 384, 326 (1996); 10.1063/1.51553

Improvements in measuring the direction to gammaray bursts with BATSE

AIP Conf. Proc. 280, 709 (1993); 10.1063/1.44256

BATSE observations of gammaray bursts

AIP Conf. Proc. 265, 13 (1991); 10.1063/1.42813 


\title{
Using BATSE to Measure Gamma-Ray Burst Polarization
}

\author{
M. McConnell*, D. Forrest* , W.T. Vestrand* and M. Finger ${ }^{\dagger}$ \\ * University of New Hampshire, Durham, New Hampshire 03824 \\ ${ }^{\dagger}$ USRA at NASA/MSFC, Huntsville, Alabama 35812
}

\begin{abstract}
We describe a technique for measuring the polarization of hard Xrays from $\gamma$-ray bursts based on the angular distribution of that portion of the flux which is scattered off the top of the Earth's atmosphere. The scattering cross section depends not only on the scatter angle itself, but on the orientation of the scatter angle with respect to the incident polarization vector. Consequently, the distribution of the observed albedo flux will depend on the direction and the polarization properties (i.e., the level of polarization and polarization angle) of the source. Although the BATSE design (with its large field-of-view for each detector) is not optimized for albedo polarimetry, we have nonetheless investigated the feasibility of this technique using BATSE data.
\end{abstract}

\section{INTRODUCTION}

One of the fundamental properties of electromagnetic radiation is its polarization. Although this property has been exploited extensively for astrophysical studies at longer wavelengths (e.g., radio, optical and, to some extent, $\mathrm{x}$-ray wavelengths), there have been only limited efforts to measure polarization from cosmic sources at hard X-ray energies $(E>30 \mathrm{keV})$. Here we investigate how one might use the radiation which is backscattered from the Earth's atmosphere to study source polarization, a technique which we call albedo polarimetry. Based on our Monte Carlo simulations, we have estimated the BATSE sensitivity to hard X-ray polarization in $\gamma$-ray bursts and find significant sensitivity to polarization for events whose $(50-300 \mathrm{keV})$ fluence is greater than $\sim 10^{-5}$ ergs $\mathrm{cm}^{-2}$.

All studies of $\gamma$-ray bursts to date have involved measurements of the source location, source spectrum and source intensity. Since these data have not yet yielded any fundamental answers as to the nature of the bursts, we contend that an additional piece of information is desirable. The only additional piece of information which is available to the observer is that of the polarization of the burst radiation. Although the published literature on the subject of polarization in bursts is limited (driven, in part, by a lack of observations), there appears to be a general consensus that such measurements would be useful $(1,2)$. In principle, polarization measurements can provide information on both the emission mechanism and on the geometry of the emitting region.

(C) 1996 American Institute of Physics 


\section{X-RAY ALBEDO POLARIMETRY}

A measurement of the linear polarization of a transient event (such as a $\gamma$-ray burst) can, in principle, be made by measuring the angular distribution of the albedo flux, i.e., the source flux which is scattered from the Earth's atmosphere prior to reaching the detector. This concept is based on the properties of the Compton scattering of polarized radiation. In particular, this approach relies on the fact that, in the case of linearly polarized radiation, the scattered photon tends to be ejected at right angles to the electric field vector of the incident radiation (3). The atmosphere, as seen from an orbiting satellite, presents a wide range of possible scatter angles for a given source direction. The photon scatter angle will depend on look direction. Hence, the intensity distribution of the albedo flux will exhibit an angular distribution which will depend on the polarization properties of the source radiation.

For the case of an orbiting detector observing some cosmic source, the distribution of the scattered albedo flux across the top of the atmosphere will depend on three parameters: 1) the angular height of the source above the Earth's limb (i.e., the source-earth-detector geometry); 2) the level of linear polarization of the source flux; and 3) the orientation of the bulk polarization vector of the source flux. Therefore, if one knows the direction of the source, then the polarization properties can be determined from the study of the albedo flux.

We have modeled the scattering process using Monte Carlo simulations, incorporating a version of GEANT which has been modified to handle polarized photons. For an incident $\mathrm{E}^{-2}$ power-law spectrum, the energy distribution of the scattered flux (integrated over $2 \pi$ steradian) peaks in the $50-100 \mathrm{keV}$ range. For sources near the zenith, the albedo fraction reaches a maximum value of about $40 \%$. An example of the spatial distribution of the scattered flux, based on our simulations, is shown in Figure 1. This figure shows the intensity of the scattered flux across the visible disk of the Earth (which subtends a half-angle of $\sim 70^{\circ}$ in low Earth orbit) for the case of a $100 \%$ polarized source at a zenith angle of $0^{\circ}$.

These simulations serve to demonstrate that, in principle, one could study burst polarization by mapping out the observed distribution of the flux which is scattered off the atmosphere. A mapping of this distribution at a resolution of a few degrees would certainly provide sufficient detail for such an albedo polarimeter. Unfortunately, we have no way of mapping the albedo flux with such high precision at the present time. However, the BATSE detectors on CGRO (in particular, the Large Area Detectors, or LADs) are capable of providing a crude map of the distribution of the albedo flux. Despite the aliasing effects amongst the detectors, this capability is sufficient for measuring polarization parameters both in some of the larger $\gamma$-ray bursts and in some of the larger solar flare events (4). 


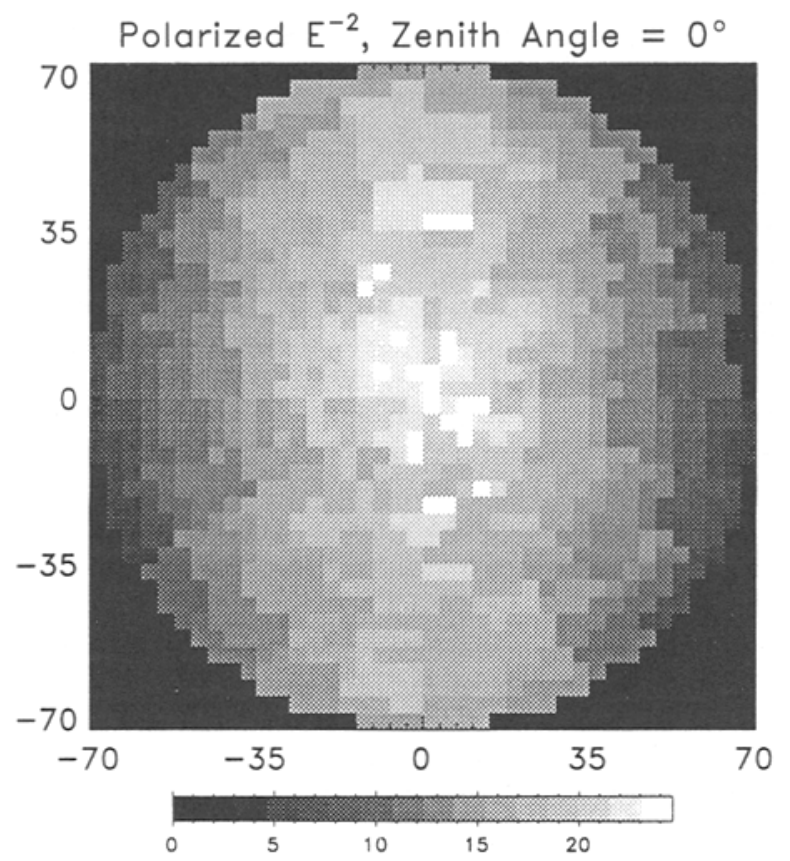

FIG. 1. The albedo distribution corresponding to a $100 \%$ polarized $\mathrm{E}^{-2}$ spectrum incident from $0^{\circ}$ zenith angle. The polarization (E-field) vector lies along the horizontal plane. The scale is a relative measure of the photon flux from each solid angle element. The asymmetry of the distribution is especially apparent near the Earth's limb.

\section{BATSE SENSITIVITY TO BURST POLARIZATION}

The exact sensitivity of the BATSE to the measurement of burst polarization depends on several factors. These include the following:

1) Source zenith angle. The sensitivity will depend on the contrast amongst the different LAD detectors. This depends not only on the contrast within the polarized flux distribution, but also on the contrast (or lack thereof) in the corresponding unpolarized distribution. These effects are such that the sensitivity is near maximum for source zenith angles $\sim 30^{\circ}$.

2) Aliasing. Due to the large FoV and the geometrical spacing of the LAD detectors, there exist certain geometrical arrangements with respect to the polarization vector where the polarization sensitivity will be limited 
(and perhaps even go to zero). In more general terms, the polarization sensitivity will vary within the polarization parameter space.

3) Incident source spectrum. Because the scattered albedo photons tend to result from incident photons $>100 \mathrm{keV}$, a harder incident spectrum will result in more intense albedo flux and, hence, higher sensitivity. This means, for example, that there will be less sensitivity to a (typically softer) solar flare than to a (typically harder) $\gamma$-ray burst of comparable fluence.

4) Source inensity. For more intense events, various instrumental effects, most notably pulse pile-up and deadtime, become important factors.

5) Flux leakage. We have assumed an LAD angular response which goes to zero at $90^{\circ}$. In other words, we assumed that the leakage of flux through the spacecraft can be neglected. (This is known not to be the case for some of the larger events and may even be the limiting factor in the application of this technique to BATSE data.)

6) Background level. The total background level for a given event depends not only on the background rate, but also on the duration of the event.

In estimating BATSE sensitivity levels, we consider only the four detectors which face away from the burst and which are exposed only to the albedo flux. We then search for a distribution of events in those detectors which are statistically inconsistent with that expected from an unpolarized event. In practice, this involves a $\chi^{2}$ comparison between the event distribution derived from albedo simulations for a polarized and an unpolarized $\gamma$-ray burst at a given zenith angle.

The estimated polarization sensitivity levels for a burst at a zenith angle of $30^{\circ}$ are shown in Figure 2. These data indicate that significant sensitivity to polarization can be achieved for bursts with (30-500 keV) fluence levels above $\sim 10^{-5} \mathrm{ergs} \mathrm{cm}^{-2}$. In the BATSE $2 \mathrm{~B}$ catalog, there were more than 50 events above this fluence level, suggesting that there may be a significant number of events which are susceptible to polarization measurements using $\mathrm{X}$-ray albedo polarimetry.

\section{BATSE MEASUREMENTS OF BURST POLARIZATION}

At the present time, we have no firm results (either positive detections or upper limits) for any $\gamma$-ray burst observed by BATSE. The analysis is presently dominated by several systematic effects which preclude any definitive measurements. These effects include: 1) differences in channel bandwidths amongst the LAD detectors; 2) deadtime effects; 3) leakage of incident flux through the spacecraft; and 4) the need to account for the precise form of the incident spectrum. The ongoing analysis is concentrating on the effort to remove these systematics effects. We are also in the process of setting up 
BATSE Polarization Sensitivity : Zenith Angle $=30$ degs

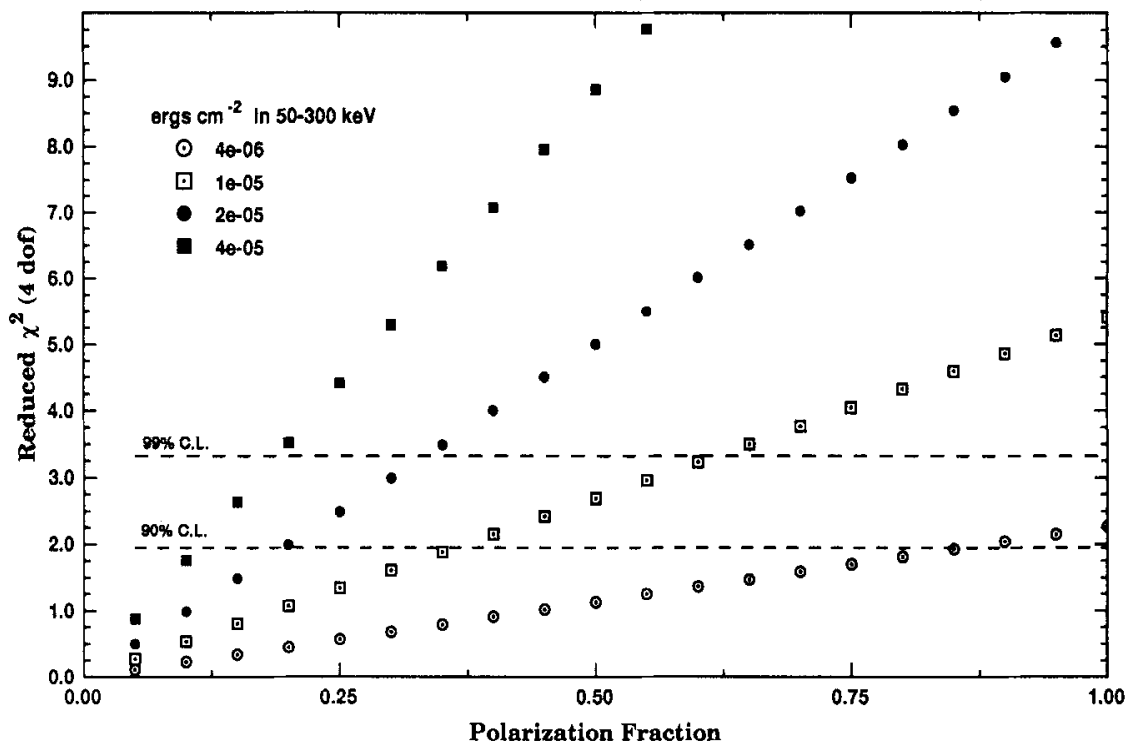

FIG. 2. Estimated sensitivity to burst polarization for an event at $30^{\circ}$ zenith angle. These data indicate, for example, that a polarization level of $\sim 70 \%$ can be detected at the $3 \sigma$ level in a burst with a fluence level of $10^{-5}$. At a fluence level of $4 \times 10^{-5}$, the $3 \sigma$ polarization sensitivity level is $\sim 15 \%$.

our simulation database in a form that can be directly used by software tools developed by the BATSE team. This should greatly facilitate the search for burst polarization in the BATSE database.

Acknowledgements: This work is supported at UNH by NASA Grant NAG 5-2388 and by NASA contract NAS 5-26645.

\section{REFERENCES}

1. K. Hurley, in Gamma-Ray Bursts: Observations, Analyses and Theories, eds. C. Ho, R. Epstein, and E. Fenimore, Cambridge: Cambridge Univ. Press, 279 (1992).

2. N. Lund, in Gamma-Ray Bursts: Observations, Analyses and Theories, eds. C. Ho, R. Epstein, and E. Fenimore, Cambridge: Cambridge Univ. Press, 489 (1992).

3. R.D. Evans, The Atomic Nucleus, New York: McGraw-Hill Book Co. (1955).

4. M. McConnell, et al., in High Energy Solar Physics, AIP Conf. Proc., in press (1996). 